\title{
BIBLIOGRAFIA
}

\section{Indice de la Revista Taller}

\section{Advertencia Preliminar}

Taller fue una revista mensual de mucha importancia en la vida literaria de México entre I938 y I94I. No sólo figuró entre las revistas de diálogo hispanomexicano durante aquellos años de crisis, sino que también sirvió de "taller" para los jóvenes Paz, Solana y otros autores ahora bien consagrados.

Los números de la tevista aparecieton con el ritmo siguiente:

$$
\begin{aligned}
& \text { I diciembre de } 1938 \\
& 2 \text { abril de I939 } \\
& 3 \text { mayo de } 1939 \\
& 4 \text { julio de } 1939
\end{aligned}
$$

Hasta este cuarto número, los Responsables eran Octavio Paz, Rafael Solana, Efraín Huerta y Alberto Quintero Alvarez.

$$
\begin{aligned}
& \text { V octubre de I939 } \\
& \text { VI noviembre de I } 939 \\
& \text { VII diciembre de } 1939 \\
& \text { VIII-IX enero-febrero de I940 } \\
& \text { X marzo-abril de I } 940 \\
& \text { XI julio-agosto de I940 } \\
& \text { XII enero-febrero de I94I }
\end{aligned}
$$

Para los números V hasta XII había un Director: Octavio Paz; un Secretario: Juan Gil-Albert; y varios socios de redacción: 
Alvarado, José, núms. X, XI [XII?],

Huerta, Efraín, núms. V, VI, VII, [VIII-IX?] X, XI, [XII?],

Quintero Alvarez, Alberto, núms. V-XII,

Sánchez Barbudo, Antonio, núms. V-XII,

Solana, Rafael, núms. V-XII,

Varela, Lorenzo, núms. V-XII y

Vega Albela, Rafael, núms. VI, VII.

También hay que mencionar que la revista disfrutó del talento artístico de José Moreno Villa, quien contribuyó con dibujos y viñetas en varios números.

El índice de autores que sigue se ha arreglado alfabéticamente. Dentro de cada autor, la lista de artículos, poesías, etc., queda en orden cronológico. Se incluyen la paginación exacta y el primer verso de cada poesía. Al final del índice se hallará un APENDICE DE TRADUCCIONES aparecidas en la revista Taller.

JAMES K. MCDONALD

Queen's University.

\section{INDICE ALFABETICO DE AUTORES}

Abréu Gómez, Ermilo, "¡Los enemigos del teatro!", 4 (julio, I939), 55-56 [Sobre Enrique Díez-Canedo].

Alberti, Rafael. "Del pensamiento en un jardín", XII (enero-febrero, I94I), 5-IO.

Alvarado, José, "Antonio Machado", 3 (mayo, I939), 23-29.

—., "Una historia del estilo francés", V (octubre, r939), 64-66. [Reseña sobre Alberto Thibaudet, Historia de la Literatura Francesa, Buenos Aires: Losada, I939].

—_, "Pensamiento y poesía", VII (diciembre, I939), 52-55 [Reseña sobre María Zambraon, Pensamiento y Poesía en la Vida Española, México: Casa de España en México, I939].

_-, "La evolución política de México", XI (julio-agosto, I940), 6264.

Arai, Alberto T., "Música y Cine", 3 (mayo, I939), 51-53. 
Barreda, Octavio G., traductor, Eliot, T. S., "Un canto para Simeón", X (marzo-abril, r940), 90-9r.

Beltrán, Neftalí, "Poemas", XI (julio-agosto, I940), 37-40 [Son los siguientes: "Soledad enemiga", págs. 37-40, y

"Soneto: Hoy me pondré a llorar por no mirarte", pág. 40].

Bergamín, José, "Siete sonetos impuntuales", 4 (julio, r939), r7-2I. [Son:

"Una explosión de soles deposita", "AAquí para morir me falta vida!"

"Vayamos a decirle al alba clara",

"Parece que se acerca, cada día",

"Al sentir que en mis ojos reaparece",

"No te perdí de vista, ni de oído", y

"Dios sabe dónde encuentra el alma asilo"].

—.'Las pequeñeces del monio", VI (noviembre, r939), 5-18.

—, "Las cosas claras", VIII-IX (enero-febrero, I940), 53-55 [Palabras pronunciadas en la Conferencia Panamericana de Ayuda a los Republicanos Españoles"].

Cabada, Juan de la, "María 'la voz'", VI (noviembre, 1939), 24-43. Cardoza y Aragón, Luis, "Soledad de la fisiologia", V (octubre, r939), 2I-26 [Poema].

—_, "Demagogos de la poesía", VIII-IX (enero-febrero, I940), 47-50.

- "Breve tratado de los cuernos de Anfitrión", XI (julio-agosto, I940), 27-36.

-, "Flor y fruto y flor nuevamente. Textos y pretextos por Xavier Villaurrutia", XI (julio-agosto, I940), 73-77 [Reseña sobre Xavier Villaurrutia, Textos y Pretextos, México: Edicionse de la Casa de España en México, r940].

Carrillo de Sotomayor, Luis [1583-16ro], "Poesías de Luis Carrillo de Sotomayor", VIII-IX (enero-febrero, I940), 73-96 [Introducción y selección de Pedro Salinas, empleando texto y notas de Dámaso Alonso, Madrid: Ed. Signo, r936. Precedidas de Quevedo, Francisco de, "Canción fúnebre a la muerte de don Luis Carrillo de Sotomayor". Esta selección consta de los sonetos siguientes: 
"El ejemplo de las cosas que fueton y se acabaron", pág. 79,

"A la ligereza y pérdida del tiempo", pág. 79,

"A la duración de un pensamiento", pág. 80,

"A un chopo, semejante en desgracia a su amor", pág. 80,

"A su amor, en sus males sin remedio", pág. 8r,

"En el sepulcro de una dama", pág. 8I,

"A las penas del amor inmortales", pág. 82,

"A las prisiones del amor, imposible [sic] de romper", pág. 82, del romance:

"A la caza de una galeotas turquescas", págs. 83-84, y del poema:

"Fábula de Acis y Galatea", págs. 85-94].

Castro Leal, Antonio, "Arte y moral de Alarcón" V (octubre, 1939), 13-20 [Fragmentos de su libro sobre la vida y la obra de don Juan Ruiz de Alarcón].

—, editor "[Don Juan Ruiz de Alarcón] Fragmentos sobre el Amor y las Mujeres", V (octubre, r939), 67-89 [Selección y prólogo, págs. 69-72, de Antonio Castro Leal].

-, "El cazador del ritmo universal", VIII-IX (enero-febrero, I940), I6-24 [Cuento].

—. "Horacio en español", VIII-IX (enero-febrero, I940), $56-58$ [Reseña sobre Horacio, Odas y épodos, prólogo y edición de María Rosa Lida, Buenos Aires: Losada. "Las cien obras maestros de la literatura y del pensamiento universal"

Cernuda, Luis, "Atardecer en la catedral", X (marzo-abril, I940), I517 [Poema].

Cruz, Sor Juana Inés de la, [I65I-I695], "Endechas de Sor Juana Inés de la Cruz (r65I-r695)", VII (diciembre, r939), 59-89 [Edición $y$ notas de Xavier Villaurrutia].

Ceusta, Jorge, "Sonetos", X (marzo-abril, 940), I8-20 [Son los siguientes:

"La mano explora en la frente", pág. 18 ,

"Hora que fué, feliz, aun incompleta", pág. I9,

"Fué la dicha de nadie ésta que huye", págs. I9-20, y

"La flor su oculta exuberancia ignora", pág. 20].

—, traductor, Spender, Stephen, "Sin el afán que da a una vida entera [soneto]", X (marzo- abril, I940), 2I. 
Díez-Canedo, Enrique, "Antonio Machado. Poeta español", 3 (mayo, I939), 7-I6.

Felipe, León, traductor, Eliot, T. S., "Los hombres huecos", X (marzoabril, 1940), 85-88.

_., "El Gran Responsable (Grito y salmo)", XI (julio-agosto; 1940), 5-26.

Ferrel, José traductor, Rimbaud, Juan Arturo, "Temporada de infierno", 4 (julio, I939), 7-37 [Paginación independiente. Esta traducción viene precedida por una nota preliminar sobre "Rimbaud". por Luis. Cardoza y Aragón, págs. 3-5].

Flores, Angel, traductor, Eliot, T. S., "Tierra baldía", X (marzo-abril, I940), 7r-84 [Hay notas sobre este poema ne las págs. roo-I05].

García Lorca, Federico, "Poemas", I (diciembre, r938), 33-50 [Son:

"Pequeño vals vienés", págs. 34-35,

"La suite del agua", págs. 36-40,

"Cuatro Baladas Amarillas", págs. 40-44,

"La selva de los relojes", págs. 44-48, y

"Herbarios", págs. 48-50].

Gaya, Ramón, "Pintura francesa contemporánea", V (octubre, r939), 49-52.

—_, "Sonetos de un diario", VII (diciembre, I939), 23-26 [Son:

$$
\begin{aligned}
& \text { I “A una verdad", pág. } 23, \\
& \text { II "Al silencio", pág. 24, } \\
& \text { III "Al sufrimiento", pág. 24, } \\
& \text { IV "A Dios", pág. 25, } \\
& \text { V "A la lámpara", pág. 25, y } \\
& \text { VI "A mis amigos", pág. 26]. }
\end{aligned}
$$

—- "Desmaño y justeza de Mariano Orgaz", VIII-IX (enero-febrero, I940), 5I-52 [Una apreciación del pintor y arquitecto español Mariano Orgaz].

—., "El señor Domenchina", X (marzo-abril, I940), 57-59.

—_, "Palabras de Despedida para mis Compeñeros de Redacción", XI (julio-agosto, 1940), 81-82. 
Gil-Albert, Juan, "A los sombreros de mi madre y otras elegias", 2 (abril, r939), 4r-62 [Estas elegías en prosa poética llevan los títulos siguientes:

"Elegía a los sombreros de mi madre", págs. 43-45,

"Elegía a la revelación de mi génesis", págs. 46-47,

"Elegía a Chenonceaux", págs. 49-50,

"Elegía a un secreto", págs. 51-52,

"Elegía a un efímero abrazo", págs. 53-54,

"Elegía a mis manos de entonces", págs. 55-56,

"Elegía a una tarde purisima (homenaje a Lucrecio)", págs.

57-62. La página 62 lleva la fecha de 1934].

- , "En torno a la Vocación (Lo popular y lo social)", 3 (mayo, I939), 54-56 [Esta prosa lleva la fecha de Cataluña, r938].

—, "Meditaciones españolas", VI (noviembre, I939), 48-55 ["Estas breves prosas escritas sobre un álbum de guerra, se publican sin la segunda meditación que llevaba por título: 'Los hombres' "]. —., "Los ídolos", VII (diciembre, I939), 37-40 [Poema].

J. G. A. [Juan Gil-Albert?], traductor, Hölderlin, Federico, "Del 'Hyperion' ", X (marzo-abril, r940), 5-9.

Gil-Albert, Juan, "Imprecación a una divinidad hostil", X (marzo-abril, 1940), 30-36 [Poema].

—, "Emilio Prados de la 'Constelación Rosicler'", XI (julio-agosto, I940), 68-7x.

Giner de los Ríos, Francisco, "Poemas", VI (noviembre, 1939), 44-47 [Son:

"Tan cerca de vosotros que el aire se conmueve", págs. 44-45.

"Presencia de España", pág. 46, y

"A tu honda memoria", págs. 46-47].

González Rojo, Enrique, "Elegías Romanas", 4 (julio, I939), 30-35 [Son nueve poesías fechadas en México, 1935].

Guerrero, Enrique Gabriel, "Poemas", 3 (mayo, 1939), 17-20 [Son:

"Preludio breve ,págs. 17-18,

"Música de cámara", págs. I8-r9, y

"Ejercicio de estío", págs. r9-20]. 
—, "Condición esforzada" [y], "Ocios de estío", XII (enero-febrero, I94I ), 48-49-50-5I.

Henestrosa, Andrés, "Retrato de mi madre", I (diciembre, 1938), 2332.

Hernández, Efrén, "Sobre causas de títeres", 2 (abril, 1939), 5-ro.

—, "El ángel del subsuelo", XII (enero-febrero, I94I), 52-58.

Herrera Petere, José, "iQué encantadora fiesta!" V (octubre, r939), 27-35.

—, "Oportunidad de Gil Vicente", X ((marzo-abril, I940), 53-54 [Reseña sobre Poesias de Gil Vicente, ed., notas y prólogo de Dámaso Alonso, México: Ed. Séneca, I940].

Huerta Efrain, "Poemas", I (diciembre, 1938), I4-22 [Son:

"Verdaderamente", págs. I4-I7,

"La poesía enemiga", págs. I7-20,

"Breve canto", pág. 2c, y

"Cuarto canto de abandono", págs. 20-22].

E. H. R. [Efraín Huerta R.?], "Tres libros españoles", I (diciembre, I938), 60-63 [Reseña de:

Serrano Plaja, Arturo, El bombre y el trabajo, Barcelona: Ediciones "Hora de España", junio de I938,

Sánchez Barbudo, Antonio, Entre dos fuegos. Narraciones (19371938), Barcelona: Ediciones "Hora de España", junio de 1938, $\mathrm{y}$

Gil-Albert, Juan, Son nombres ignorados... elegías, sonetos, Himnos, Barcelona: Ediciones "Hora de España", I938].

Huerta, Efraín, "Tramontar", 4 (julio, 1939), 43-47.

—- "Preesncias", 4 (julio, r939), 54-55 [Sobre José Bergamín].

—_, "Problema del alma", VIII-IX (enero-febrero, I940), I2-I5 [Poema].

—-, "Organización del sarcasmo", XI (julio-agosto, I940), 7I-73 [Sobre Herrera Petere, José, Niebla de Cuernos (Entreacto en Europa), México: Ed. Séneca, I940. Col. Lucero].

—_, "Problema del alma", XII (enero-febrero, I94I), 46-47 [Poema]. 
—, "Una antología de forcejeos", XII (enero-febrero, I94I), 68-70 [Sobre Maples Arce, Manuel, editor, Antología de la poesía mexicana moderna, Roma: Poligráfica Tiberiana, I940].

—, "La nube exacta y el reloj nublado", XII (enero-febrero, r94I), 70-7.x [Sobre Cardoza y Aragón, Luis, La nube y el reloj (Agustín Lazo, Carlos Mérida, Rufino Tamayo, Julio Castellanos, David Alfaro Siqueiros, Diego Rivera, José. Clemente Ōrozco), México: U.N.A.M., I940].

Ibáñez, Sara de, "Versos de Sara de Ibáñez", XII (enero-febrero, I94I), 34-42 [Son:

"Liras", págs. 34-38, y

"Sonetos":

"Semillero de soles y azucenas", pág. 39,

"Mi boca dio una flor para abolirse", págs. 39-40,

"Traspasé las fronteras de la rosa", págs. 40-4r,

"Lengua del mal guijarto de la muerte", pág. 4I, e

"Isla en la tierra: Al norte el frío y su jazmín quebrado", pág.

42. La selección leva un introducción de Pablo Neruda, pág.

34].

Jiménez, Juan Ramón, "Los árboles", X (marzo-abril, I940), IO-I I [Poema de 38 versos, de los cuales el prmiero es: "Volv'a yo con las nubes"].

-, traductor, Eliot, T. S., "La fligia [sic] che piange", $\mathrm{X}$ (marzoabril, I940), 70.

—, traductor, Eliot, T .S., "Marina", X (marzo-abril, I940), 89.

Landsberg, Pablo Luis, "Piedras blancas", VIII-IX (enero-febrero, 1940), 5-II ["Del libro La experiencia de la muerte próximo a publicarse en las Ediciones Séneca". Prosa].

J. L. [Juan Larrea?]" [Ponce, Aníbal] Humanismo burgués y bumanismo proletario [México: Editorial América, 1938]", I (diciembre, 1938), 63-64.

Larrea, Juan, "Poesía a vistad e pájaro", X (marzo-abril, I940), 54-57 [Notas sobre "Piedra y cielo", págs. 54-56, los cuatro primeros "cuadernos de poesía", colección colombiana dirigida por Jorge Ro- 
jas, y "El ciervo herido", págs. 56-57, colección poética de Manuel Altolaguirre].

Machado, Antonio, "Del camino", X (marzo-abril, r940), 39-40 [Poesía de 36 versos, que comienza: "¿Oh, dime, noche amiga, amada. vieja", y que lleva esta nota de introducción: "Como recuerdo, Taller publica este hermoso fragmento de Antonio Machado muerto. hace un año, en la emigración, junto a los que, con un heroísmo. ejemplar habían defendido contra el extranjero, el suelo y la luz de su. patria"].

Magaña Esquivel, Antonio "[Moreno Villa, José], Cornucopia de Méxi-. co [México: Ediciones de la Casa de España en México, I940]" XI (julio-agosto, 1940), 80-8I.

Martínez, José Luis, "Julio Torri", XII (enero-febrero, I94r), 66-68. Moreno Villa, José, "oTpografía de la casa paterna. (Visión supersticiosa)", VII (diciembre, I939), 27-36 ["Fragmento" de prosa].

Neruda, Pablo, editor, "Liras de Luis Martín, Sor Juana Inés de la Cruz, el conde de Bebolledo, doña Cristobalina y el conde de Villamediana", VI (noviembre, I939), 69-9r [Selección y discurso, en verso, de las liras por Pablo Neruda].

—_, "Versos de Sara de Ibáñez", XII (enero-febrero, I94I), 34-42 [Introducción de Pablo Neruda, pág. 34].

Ortiz de Montellano, Bernardo, editor, Eliot, T. S., "Poemas", X (marzo-abril, I940), 6I-I05 ["Nota previa" de Ortiz de Montellano, págs. 63-64].

—, traductor, Eliot, T. S., "Miércoles de ceniza", X (marzo-abril, I940), 92-99.

Palacios, Emmanuel, "Paisajes y confesiones", 4 (julio, I939), 48-50 [Prosas fechadas en México, 1935-I939].

Paz, Octavio, "Vigilias (Fragmentos del diario de un Soñador)", I (diciembre, 1938), 3-I3.

O. P. [Octavio Paz?], "La casa de España", I (diciembre, I938), 5758.

Paz, Octavio, "Razón de ser", 2 (abril, r939), 30-34 [Prosa].

- -, "El mar (Elegía y esperanza)", 3 (mayo, I939), 4I-44 [Prosa]. 
—, "Oda al sueño", 4 (julio, I939), 36-39 [Primer verso: "Cerca de mí te escucho"].

—_, "Constante amigo", 4 (julio, I939), 53 [Sobre Emilio Prados]. - et. al., "Tarjetas", 4 (julio, 1939), 56.59 [Informes sobre los recién llegados de España].

—-, "Una obra sin joroba", V (octubre, I939), 43-45 [Prosa].

-_, "Invitación a la novela", VI (noviembre, I939), 66-68.

I939), II-22 [Prosa].

-_, "Noche de resurrecciones (Fragmentos)", X (marzo-abril, r940), 25-29 [Poema en tres partes].

seña sobre Ballagas, Emilio, Sabor eterno].

-.., "Mundo de perdición", XI (julio-agosto, 1940), 65-68.

—_, "Silvestre Revueltas", XII (enero-febrero, I94I), 6I-63.

-_. "Las 'Páginas escogidas' de José Vasconcelos", XII (enero-febrero, I94I), 64-66.

Pellicer, Carlos, "Sonetos", VII (diciembre, I939), 5-10 [Son:

"Sonetos de otoño" A Luis Barragán:

I "Primer cielo de otoño, primer vuelo", pág. 5,

II "Pausa de otoño, poderosa y lenta", pág. 6, y

III "Aquí, rayando sus cristales fríos", págs. 6-7,

"A Eduardo Villaseñor, enviándole una rara cactácea":

"Al aire serpentín de esta figura", pág. 7, fechado en Las Lomas, octubre I I de I939, y

"Sonetos de los arcángeles" A José Bergamin:

"San Gabriel", pág. 8, "San Miguel", pág. 9, y "San Rafael", págs. 9-1o, fechados en México, D. F., abril o mayo de 1937$]$.

—-, "Elegía Nocturna", X (marzo-abril, I940), I2-I4 [Poema en cuatro partes, fechado en México, D. F., a 26 de diciembre de 1939]. A. P. [Aníbal Ponce?], "Fourier", VIII-IX (enero-febrero, I940), 69- 
72 [Sobre Armond y Maubliac, Fourier. México: Fondo de Cultura Económica, I940, Serie de Los Inmortales].

Prados, Emilio, "Cuerpo perseguido", 4. (julio, I939), 22-28 [Poema en nueve partes, fechado I928-1929].

—, "Canto a la soledad", X (marzo-abril, r940), 22-24 [Poema].

Quintero Alvarez, Alberto, "Sobre la inteligencia", I (diciembre de r938), 54-56.

_-, "El tiempo contemplado", 2 (abril, I939), II-I4 [Poema en cuatro partes. Advierte una nota, pág. II, que la primera parte ya apareció en Las cien mejores poesías mexicanas modernas, selección de Antonio Castro Leal, México: Porrúa, 1939].

—., "La fatalidad en Kafka", 2 (abril, I939), 37-39 [Se trata de Kafka, Franz, La Metamorfosis, trad. directa del alemán, y prólogo, por Jorge Luis Borges, Buenos Aires: Ed. Losada].

- . "El poeta León Chestov", 3 (mayo, 1939), 39-4x.

—-, "Enrique González Rojo", 4 (julio, r939), 29 [Introducción a Las Elegias Romanas de González Rojo, q. v.]

—, "Los inquilinos de la filosofía", V (octubre, 1939), 45-49 [Prosa].

_., "Estancias", VI (noviembre, I939), I9-23 [Son:

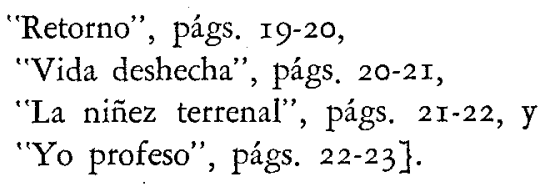

-., "Un gran poeta y su obra", VII (diciembre, I939), 44-46 [Sobre Gorostiza, José, Muerte sin fin, México: Ediciones R. Loera y Chávez, 1939].

—, "En el mar de Platón", VIII-IX (enero-febrero, 1940), 62-64 [Sobre Zambrano, Maria, Filosofía y Poesía, Morelia: Publicaciones de la Universidad Michoacana].

—., "La otra vida en Pirandello", XI (julio-agosto, 1940), 77-79 [Sobre Pirandello, Luis, La vida que te di, tr. por Agustín Lazo y X. V., México: Ediciones Nueva Cultura, Tomo I, Núm. 4, I 40$]$. --, "Carta al amigo que vive junto al mar", XII (enero-febrero, 194r), 43-45 [A Enrique Gabriel Guerrero]. 
—., "[Abréu Gómez, Ermilo], Canek [México, r940]", XII (enerofebrero, I94I), 74 .

—, "Claro abismo", XII (enero-febrero, I94I), 75.

Rejano, Juan, "Escala de la ausencia", VIII-IX (enero-febrero, r940), 25.27 [Poema en cinco partes].

—, "Vallejo entre el clamor y el silencio", X (marzo-abril, r940), 47-5I [Sobre Vallejo, César, España, aparta de mi este cáliz, México: Ed. Séneca, 1940. Col. Lucero].

J. R. [José Revueltas?], "La iglesia y el hombre", r (diciembre, 1938), 5I-53. Revueltas, José, "El Quebranto (capítluo primero de una novela en prensa)", 2 (abril, I939), I5-27.

—, "Antonio Machado", 2 (abril, I939), 28 [Nota sobre la muerte del poeta].

——, "Profecía de España", 2 (abril, 1939), 28-30 [Prosa].

—. "La 'Carta a un Amigo Difunto'", 4 (julio, I939), 5r-52 [Nota sobre la muerte del poeta Guitérrez Hermosillo].

Reyes, Alfonso, "Unna de Alarcón", V (octubre, I939), 7-Ix [Prosa]. Ruiz de Alarcón y Mendoża, don Juan [I581-I639], "Fragmentos sobre el Amor y las Mujeres", V (octubre, I939), 67-89 [Selección y prólogo, págs. 69-72, de Antonio Castro Leal].

Salinas, Pedro, editor, Carrillo de Sotomayor, Luis, "Poesías de Luis Carillo de Sotomayor", VIII-IX (enero-febrero, I940), $79-36$ [Introducción y selección de Pedro Salinas, empleando texto y notas de Dámaso Alonso, Madrid: Ed. Signo, 1936. Precedidas de Francisco de Quevedo, "Canción fúnebre a la muerte de don Luis Carrillo de Sotomayor].

Sánchez Barbudo, Antonio, "La maravilla en la sangre", V (octubre, I939), 53-60 [Reseña de Crónicas de la conquista (antología), ed. Agustín Yáñez, México: U.N.A.M. Biblioteca del Estudiante Universitario, 2].

—-, "Sobre la novela de un poeta", VII (diciembre, 1939), 46-52 [Reseña de Alain Fournier, El gran Meaulnes, Buenos Aires: Sur, 1938].

-, "[Felipe, León], El español del éxodo y del llanto", VIII-IX (enero-febrero, r940), 58-6r.

-, "Primavera-Otoño, I938", XI (julio-agosto, I940), 4I-6I [En la pág. 6r, dice: "concluirá"]. 
_. "Antonio Machado", XII (enero-febrero, I94I), II-33.

Sánchez Vázquez, Adolfo, "Sonetos", XII (enero-febrero, I94I), 59-60 [Son:

I "¡Oh, tronco adolescente y sin sabores", pág. 59,

II "Tú soledad empieza a estremecerme", págs. 59-60, y

III "No quiere que derrames tu lamento", pág. 60]

Solana, Rafael, "Una acuarela y viñetas de Maria Izquierdo", I (diciembre, 1938), dieciséis páginas sin numerar entre 32 y 33.

-. "[Castro Leal, Antonio, editor], Las cien mejores poesias mexicanas modemas [México: Porrúa, 1939]", 2 (abril, I939), 34-47.

_- "Diario, epígrafes y apuntes para la novela La Educación de los Sentidos (Ediciones Taller, r939)", 3 (mayo, 1939), 44-50.

_-., "Tarjetas", 3 (mayo, 1939), 57-58 [Notas varias].

_- "Dos poemas", 4 (julio, I939), 40-42 [Fechados en Argel, mayo de I939, para Octavio Paz].

—_, "En busca de la prosa", VI (noviembre, I939), 63-65.

—_, "Capítulo IV", VIII-IX (enero-febrero, I940), 28-4I [De la novela en preparación La mujer de sal. Con fecha de Roma-Capri, I939].

R. S. [Rafael Solana?], “Nueva Cvltvra!”, XIII-IX (enero-febrero, 1940), 68-69 [Sobre la colección "Nueva Cvltvra" de Xavier Villaurrutia, de la Editorial "Cvltvra"].

—- "Ricardo E. Molinari", X (marzo-abril, I940), 46 [Nota sobre Libro de las Soledades del Poniente y La corona, ambos publicados en Buenos Aires: Francisco A. Colombo].

Toscano, Salvador, "Las ideas políticas de Ramón López Velarde", 3 (mayo, I939), 3I-38.

Unamuno, Miguel de, traductor, Leopardi, Jacobo, "La retama (La Ginestra)", XI (julio-agosto, I940), 83-I03.

Usigli, Rodolfo, traductor, Eliot, T. S., "El canto de amor de J. Alfred Prufrock", X (marzo-abril, I940), 65-69.

Varela, Lorenzo, "El héroe (Fragmento)", V (octubre, I939), 37-4I [Poema].

_.., "Las astillas olorosas de 'Tala", V (octubre, 1939), 60-63 [Nota crítica]. 
—., "Noviembre en la esperanza", VII (diciembre, I939), 4I-43 [Prosas].

—_, "La flauta y el pito; el tambor y el salmo; y la poesía (En torno a Rafael Alberti)", X (marzo-abril, I940), 4I-45 [Sobre Alberti, Rafael, Poesía, Buenos Aires: Ed. Losada, 1939].

L. V. [Lorenzo Varela?], "[Cabada, Juan de la], Paseo de mentiras", XII (enero-febrero, I94I), 7 I-73.

Varela, Lorenzo, traductor, Baudelaire, Charles, "Trozos escogidos de los Diarios intimos y de los Consejos a los literatos jóvenes", XII (enero-febrero, I94I), 77-94 [Selección, traducción y prólogo de Lorenzo Varela].

Varios, "Liras de Luis Martín, Sor Juana Inés de la Cruz, el conde de Rebolledo, doña Cristobalina y el conde de Villamediana", VI (noviembre, 1939), 69-9I [Selección, y discurso en verso de las liras, por Pablo Neruda].

Vega Albela, Rafael, "Dos Poemas", 3 (mayo, 1939), 2 I-22. [Son:

"Amorosamente", pág. 2I, y

"Sólo el hombre", págs. 21-22].

-—, "La luz en el destiero", VII (diciembre, 1939), 55-57 [Sobre los intelectuales españoles].

—, "Poemas", VIII-IX (enero-febrero, I940), 42-45 [Son:

Canciones: "Al sueño", pág. 42,

"A la memoria", págs. 42-43, y

Sonetos: I "Soy tu pobre, Señor, y tu desnudo", pág. 43,

II "Si con los pies hundidos en el sueño", pág. 44,

III "Amor abrió la sombra fugitiva", págs. 44-45, y

IV "Lanza clavada del dolor más hondo", pág. 45].

—, "Poesía y profecía de Jorge de Lima", VIII-IX (enero-febrero, I940), 65-67 [Nota crítica].

X. V. [Xavier Villaurrutia?] "[Shelley, Percy, B.], Adonais [México:

Editorial Polis, I938]", I (diciembre, r938), 59-60. 
Villaurrutia, Xavier, "Amor condusse noi ad una morte", 4 (julio, I939), I5-I6 [Poema].

—_, "Introducción al rigor", VI (noviembre, I939), 56.59 [Reseña de Thierry Maulnier, Introduction à la poésie française, París: Gallimard N.R.F., r939].

- editor, "Endechas de Sor Juana Inés de la Cruz (I651-I695)", VII (diciembre, r939), 59-89.

Zambrano, María, "Poesía y filosofía", 4 (julio, I939), 5-I4 [Capitulo del libro en preparación: Filosofía, Poesía y Tragedia].

—. "Descartes y Husserl", VI (noviembre, I939), 59-62 [Sobre Romero, Francisco, Descartes y Husserl. Escritos en honor de Descartes, La Plata, I938].

\section{APENDICE DE TRADUCCIONES}

Baudelaire, Charles, "Trozos escogidos de los Diarios íntimos y de lus Consejos a los literatos jóvenes", XII (enero-febrero, I94I), 77-94 [Selección, traducción y prólogo de Lorenzo Varela].

Eliot, T. S., "Poemas" X (marzo-abril, I940), 6r-I05 [Nota preliminar de Ortiz de Montellano. Son los siguientes:

"El canto de amor de J. Alfred. Prufrock", trad. de Rodolfo Usigli, págs. 65-69,

"La fligia che piange", trad. de Juan Ramón Jiménez, pág. 70,

"Tierra baldía", trad. de Angel Flores, págs. 7I-84, con notas: págs. IOO-I05.

"Los hombres huecos", trad. de León Felipe, págs. 85-88,

"Marina", trad. de Juan Ramón Jiménez, pág. 89,

"Un canto para Simeón", trad. de Octavio G. Barreda, págs. 90-9I, y

"Miércoles de ceniza", trad. de B. Ortiz de Montellano, págs. 9299].

Hölderlin, Federico, "Del 'Hyperion'”, X (marzo-abril, r940), 5-9 ['Trad. de J. G.-A.].

Leopardi, Jacobo, "La retama (La Ginestra)", XI (julio-agostor I940),

83-To3 [Traducción de Miguel de Unamuno]. 
Rimbaud, Juan Arturo, "Temporada de infierno", 4 (julio, 1939), 7-37 [paginación independiente. Nota de Luis Cardoza y Aragón, págs. 3-5; traducción de José Ferrel].

Spender, Stephen, "Soneto", X (marzo-abril, I940), 21 [Trad. de Jorge Cuesta]. 